ORIGINAL

\title{
Avaliação do crescimento dos cascos de bovinos mestiços leiteiros criados sob regime semi-intensivo
}

\author{
Evaluation of the growth of the claws of crossbred dairy cattle raised under the \\ semi-intensive system
}

\author{
Fernando Passon Casagrande ${ }^{1^{*}}$, José Renato Junqueira Borges², Marcus Dantas Dórea ${ }^{3}$, Paulo César Amaral \\ Ribeiro Silva Correio ${ }^{4}$, Pricilla Pozzatti ${ }^{5}$,Édipo Alex Malavolta Ramão ${ }^{1}$ \\ ${ }^{1}$ Universidade Federal do Pampa (UNIPAMPA), Uruguaiana, RS, Brasil \\ 2 Universidade de Brasília (UnB), Brasília, DF, Brasil \\ ${ }^{3}$ Universidade Federal do Espírito Santo (UFES), Vitória, ES, Brasil \\ ${ }^{4}$ Universidade Federal Fluminense (UFF), Niterói, RJ, Brasil \\ ${ }^{5}$ Instituto Federal de Educação, Ciência e Tecnologia Catarinense (IFC), Araquari, SC, Brasil
}

\section{Resumo}

A dor e o desconforto causados pelas lesões podais representam uma das principais causas de claudicação para os bovinos. As perdas econômicas advindas desse problema podem atingir cifras expressivas, principalmente na pecuária leiteira. As afecções podais podem ser originadas de fatores nutricionais, genéticos e ambientais, além das diferenças de distribuição de peso entre os dígitos. Objetivou-se com o presente estudo avaliar o crescimento dos cascos de 25 animais mestiços leiteiros criados sob regime semi-intensivo. Para isso, foram feitas marcas na muralha do casco com a ajuda de uma furadeira e a medição realizada mensalmente. Os resultados encontrados indicaram taxa de crescimento médio das unhas de $5,4 \mathrm{~mm} / \mathrm{mês}$, com variações entre 4,8 e $6,3 \mathrm{~mm} / \mathrm{mês}$. Houve diferença significativa quando comparado o crescimento dos cascos dos membros torácicos e pélvicos, sendo que os últimos apresentaram um crescimento superior. As unhas laterais e a região abaxial das mesmas apresentaram um crescimento maior. As maiores taxas de crescimento foram encontradas nos cascos dos membros posteriores, assim como nas unhas laterais e, por fim, na região abaxial das unhas.

Palavras-chave: Crescimento. Podologia. Unha. Vacas de Leite.

\begin{abstract}
Lameness represents one of the main causes of pain and discomfort for cattle. The economic losses of this problem can reach significant numbers, mainly with
\end{abstract}


dairy cattle. Claw lesions may originate from nutrition, genetic and environment factors, further differences in weight distribution between digits. The aim of this study was to evaluate the hoof growth of 25 dairy cross-bred cows created under semi-intensive system. Marks were done in the wall of the hoof with the aid of a drill and the measurements taken monthly. The results found in this work indicate an average hoof growth of $5.4 \mathrm{~mm} / \mathrm{month}$, with variations between 4.8 and $6.3 \mathrm{~mm} /$ month. There was significant difference when compared the growth of the front and rear claws, having the latter grown more. The lateral hooves and the abaxial region had a higher growth. The highest growth rates were found in the hoof of the pelvic limbs, as well as in the lateral hooves and in the abaxial region of the digit.

Keywords: Growth. Podology. Claw. Dairy cattle.

\section{Introdução}

Com a intensificação dos trabalhos de melhoramento de bovinos leiteiros, progressos extraordinários começaram a surgir no que se refere a algumas características desses animais como, por exemplo, maior capacidade digestiva e respiratória, maior desenvolvimento da glândula mamária e aumento da capacidade de produção de leite. Esses resultados, entretanto, não foram acompanhados na mesma velocidade pelo melhoramento do sistema locomotor. Com isso, problemas relacionados aos membros dos bovinos adquiriram importância crescente na bovinocultura, sendo em muitos casos um dos principais entraves econômicos ao seu desenvolvimento. Ferreira et al. (2005) consideram que os problemas relativos à saúde dos cascos se constituem em uma das três principais causas de perdas econômicas, ao lado dos problemas da glândula mamária e dos problemas reprodutivos.

Nicoletti (2004) preconiza como número aceitável de animais claudicantes em um rebanho/ ano o valor de 7 a $10 \%$, mas enfatiza que esse índice é superado, e muito, nas condições brasileiras. Borges et al. (1992) observaram índices de até $14,17 \%$ de afecções podais em bovinos leiteiros submetidos a diferentes sistemas de manejo. Segundo Molina et al. (1999), o desconhecimento dessa prevalência dificulta o diagnóstico da situação e a elaboração de estratégias de controle.

Além do aspecto econômico, o efeito negativo sobre o conforto animal deve ser considerado, sendo este extremamente afetado pelos problemas de casco, ocasionando comportamentos diferenciados que visam proteger as áreas lesadas e sensíveis da pata. Dentre os principais fatores que acarretam as doenças podais, destacam-se os nutricionais, os genéticos e os ambientais, além da diferença de distribuição de peso entre os dígitos, que também é um fator importante nas alterações que levam à claudicação. Cerca de 93\% dos problemas locomotores ocorrem nos cascos. Destes, 92\% ocorrem nos membros posteriores, dos quais $68 \%$ nas unhas laterais, $12 \%$ nas unhas mediais e $20 \%$ no espaço interdigital e região periférica ao casco (Dias e Marques Jr, 2003), porém o autor não descreve as condições em que o trabalho foi realizado.

A muralha do casco cresce a partir da banda coronariana aproximadamente $5 \mathrm{~mm}(3$ a $7 \mathrm{~mm}$ ) por mês em vacas leiteiras. Na sola, a espessura média do casco é de $7 \mathrm{~mm}$ e o crescimento mensal está em torno de $3 \mathrm{~mm}$ (Ferreira et al., 2005). Segundo Hahn et al. (1986), quanto maior for o desgaste dos cascos, maior será o estímulo para o seu crescimento.

Objetivou-se com esse estudo determinar a taxa de crescimento mensal dos cascos anterior e posterior, unhas lateral e medial, e região dorsal e abaxial das unhas de bovinos leiteiros mestiços, meio-sangue Holandês/Zebu, criados sob sistema semi-intensivo.

\section{Materiais e métodos}

Para a determinação da taxa de crescimento dos cascos, foram utilizadas 25 vacas leiteiras mestiças escolhidas aleatoriamente e mantidas sob o sistema de criação semi-intensivo. Todos os animais eram mestiços de produção leiteira e pertenciam à Escola Agrotécnica Federal de Alegre (EAFA). A alimentação dos animais era à base de capim Brachiaria sp. a pasto (18 horas diárias) e suplementação com cana de açúcar e ureia (fornecida uma vez ao dia após a ordenha) quando os animais permaneciam no estábulo 
(6 horas diárias), sendo também fornecida suplementação mineral à vontade. 0 estábulo era composto de uma sala de ordenha mecânica, com piso cimentado, e área de alimentação feita com piso de paralelepípedo e coberta com sombrite, que possibilitava a entrada de sol nas instalações e, assim, diminuía o acúmulo de umidade no pátio.

Para a mensuração e cálculo do crescimento dos cascos foi empregada a técnica descrita por Hahn et al. (1986), a qual recomenda a marcação da muralha do casco em dois pontos específicos (dorsal e abaxial), em duas ocasiões diferentes. Ao invés do ferro de solda, preconizado pela técnica original, a marcação foi realizada com o emprego de uma broca de aço acoplada a uma furadeira de alta rotação. A primeira marca foi realizada no estojo córneo a um centímetro da linha do perióplo, aprofundando-se a broca três milímetros para permitir uma boa identificação, sem, no entanto, atingir o cório laminar. Cada uma das unhas recebeu duas marcações, uma dorsal e uma lateral (abaxial). As medidas foram realizadas no período de um ano, a cada 30 dias. Houve necessidade de remarcar os cascos dos animais mais duas vezes, pois à medida que as marcas se aproximavam da borda inferior da parede do casco, estas desapareciam. A segunda marcação foi realizada três meses após a primeira avaliação e a terceira, oito meses após a primeira avaliação.

As médias de crescimento dos doze meses foram utilizadas para estabelecer a taxa de crescimento dos cascos de bovinos mestiços leiteiros. Além disso, foram comparadas as médias de crescimento das unhas dos membros torácicos e pélvicos, das unhas laterais e mediais e das marcas dorsal e abaxial das unhas. Foi comparado, também, o crescimento das unhas laterais e mediais dos membros torácicos, e das unhas laterais e mediais dos membros pélvicos. Foi utilizada análise de variância para avaliar as médias gerais. No caso de significância, as médias foram comparadas, entre si, pelo teste $t$ de Student ao nível de 5\% de significância (Sokal e Rohlf, 1969).

\section{Resultados e discussão}

A comparação das médias de crescimento está apresentada na Tabela 1. Existiram diferenças quando comparados os membros torácicos e pélvicos, sendo que os últimos tiveram um crescimento superior ( $\mathrm{p}<0,009)$. 0 maior crescimento dos cascos foi verificado nas unhas laterais ( $p<0,005)$ e houve, também, maior crescimento na região abaxial da unha $(\mathrm{p}<0,0001)$.

0 crescimento médio dos cascos dos bovinos foi de $5,4 \mathrm{~mm} /$ mês, com médias variando entre 4,8 e $6,3 \mathrm{~mm} / \mathrm{mês}$, o que está de acordo com Dias e Marques Jr (2003), Nicoletti (2004) e Ferreira et al. (2005), que citam uma média de $5 \mathrm{~mm} / \mathrm{mês}, 5$ $\mathrm{mm} / \mathrm{mês}$ e 5 a $6 \mathrm{~mm} / \mathrm{mês}$, respectivamente. Silva et al. (2010) relataram um crescimento médio dos cascos de 7,2 $\mathrm{mm}$ em um período de 40 dias em vacas da raça Girolanda. Ollhoff e Ortolani (2001) encontraram um valor médio de 4,1 $4,2 \mathrm{~mm} / \mathrm{mês}$, assim como Vermunt e Greenough (1995), com valor médio de 4 a 5 mm/mês. Essas diferenças nos valores da taxa de crescimento médio dos cascos variam principalmente pelo fator abrasividade, sendo que quanto mais abrasivo é o piso, maior é o estímulo para o seu crescimento (Hahn et al., 1986).

Tabela 1 - Influência do membro, unha e região da unha sobre o crescimento dos cascos (cm/mês)

\begin{tabular}{|c|c|c|c|c|c|c|}
\hline & A & $\mathbf{P}$ & M & L & D & $A b$ \\
\hline Membro & $0,53^{a}( \pm 0,09)$ & $0,55^{\mathrm{b}}( \pm 0,10)$ & & & & \\
\hline Unha & & & $0,53^{\mathrm{a}}( \pm 0,08)$ & $0,55^{\circ}( \pm 0,10)$ & & \\
\hline Região da unha & & & & & $0,51^{\mathrm{a}}( \pm 0,08)$ & $0,57^{\mathrm{b}}( \pm 0,09)$ \\
\hline
\end{tabular}

Nota: a,b Médias seguidas de diferentes letras na mesma linha diferem estatisticamente $(\mathrm{p} \leqslant 0,05)$. A - Anterior; P - Posterior; M - Medial; L - Lateral; D - Dorsal; Ab - Abaxial. 
Vermunt e Greenough (1995) afirmaram que não existe influência racial sobre o crescimento dos cascos. Por outro lado, Murphy e Hannan (1986) compararam garrotes das raças Holandesa e Hereford, detectando maior crescimento na primeira raça. Já Ollhoff e Ortolani (2001) não encontraram diferenças significativas quando comparadas as raças Jersey e Gir. Comparando o crescimento dos cascos dos animais mestiços deste experimento com os descritos por Ollhoff e Ortolani (2001), verificamos que os animais mestiços apresentaram um crescimento superior aos encontrados nos animais das raças Jersey e Gir, porém os trabalhos foram realizados em condições de piso e de nutrição diferentes.

Segundo Clark e Rakes (1982), Tranter e Morris (1992) e Ollhoff e Ortolani (2001), não existe diferença significativa na taxa de crescimento dos cascos quando comparados os membros torácicos e pélvicos. Já Hahn et al. (1986) constataram maior crescimento nos membros pélvicos do que nos torácicos, fato que foi também confirmado neste estudo.

Encontrou-se diferença significativa ao comparar as médias da taxa de crescimento das unhas laterais e mediais, observando-se que os dígitos laterais cresceram mais do que os mediais $(\mathrm{p}<0,005)$. 0 crescimento também foi significativo quando comparada a média da taxa de crescimento nas regiões dorsal e abaxial da unha, onde foi encontrado maior crescimento na região abaxial ( $\mathrm{p}<0,0001)$. Os presentes resultados confirmaram as conclusões de Clark e Rakes (1982), Hahn et al. (1986), Distl et al. (1990) e Ollhoff e Ortolani (2001) de que ocorre crescimento maior na região abaxial da unha do que na região dorsal.

Segundo Ferreira et al. (2005), ao examinarem a distribuição de peso corporal em vacas, $60 \%$ deste peso é suportado pelos membros torácicos. Do ponto de vista anatômico, contudo, estes se inserem ao corpo através de músculos, o que tende a amortecer o impacto de peso sobre os pés, mais especificamente sobre o cório. As forças biomecânicas associadas com a variação da distribuição de peso são menos pronunciadas nos dígitos dos membros torácicos, permitindo uma menor frequência de lesões.
Esse menor impacto nos membros torácicos foi comprovado neste estudo, já que as unhas desses membros sofreram menor crescimento, o qual é proporcional ao impacto sofrido. Não houve, também, diferenças significativas no crescimento dos dígitos laterais e mediais pélvicos $(p=0,67)$, como demonstrado na Tabela 2 .

Tabela 2 - Comparação do crescimento das unhas laterais e mediais nos membros anteriores e posteriores ( $\mathrm{cm} / \mathrm{mês}$ )

\begin{tabular}{lcc}
\hline & Unhas laterais & Unhas mediais \\
\hline Membros anteriores & $0,53^{\mathrm{a}}( \pm 0,09)$ & $0,53^{\mathrm{a}}( \pm 0,08)$ \\
\cline { 2 - 3 } Membros posteriores & $0,58^{\mathrm{a}}( \pm 0,10)$ & $0,53^{\mathrm{b}}( \pm 0,08)$ \\
\hline
\end{tabular}

Nota: a,b Médias seguidas de diferentes letras na mesma linha diferem estatisticamente $(p \leqslant 0,05)$.

Os membros posteriores suportam $40 \%$ do peso do animal, porém estes se inserem ao corpo através da articulação coxo-femoral, criando uma estrutura esquelética rígida, perdendo, assim, a capacidade de diminuir os efeitos da variação de peso principalmente nas unhas laterais destes membros (Ferreira et al., 2005). Os membros pélvicos têm como principal papel impulsionar o animal para frente (Greenough e Weaver, 1997), por isso esses cascos sofrem uma sobrecarga de peso maior que a dos outros membros. Estas sobrecargas periódicas estimulam a função vascular do dígito, levando a uma maior produção de tecido córneo. Isso explica por que a maioria das afecções podais ocorre nos dígitos laterais dos membros pélvicos, como descrito por alguns autores: Russel et al. (1982) concluíram que $84 \%$ dos problemas se localizavam nos membros pélvicos, sendo 85\% nas unhas laterais; Cruz et al. (2001) relataram que 91,9\% das lesões ocorreram nas unhas laterais pélvicas; e Dias e Marques Jr (2003) aferiram que 92\% dos casos ocorreram nos membros pélvicos, dos quais $68 \%$ nas unhas laterais. No presente estudo, as unhas que apresentaram maior taxa de crescimento foram as pélvicas laterais quando comparadas às mediais ( $p<0,006)$, como demonstrado na Tabela 2 . 


\section{Conclusão}

0 crescimento médio dos cascos dos bovinos foi de $5,4 \mathrm{~mm} / \mathrm{mês}$, com maiores taxas de crescimento nos cascos dos membros posteriores, assim como nas unhas laterais e, por fim, na região abaxial das unhas.

\section{Referências}

Borges JRJ, Pitombo CA, Santiago SS, Ribeiro PN, Ronconi MA. Incidência de afecções podais em bovinos leiteiros submetidos a diferentes sistemas de manejo. Arq Esc Med Vet. 1992;15:34-42.

Clark AK, Rakes AH. Effect of methionine hydroxy supplementation on dairy cattle hoof growth and composition. J Dairy Sci. 1982;65(8):1493-1502.

Cruz C, Driemeier D, Cerva C, Corbellini, LG. Clinical and epidemiological aspects of bovine digital lesions in southern Brazil. Arq Bras Med Vet Zootec. 2001;53(6):654-7.

Dias ROS, Marques Jr AP. Casco em bovinos: identifique as lesões, as novas técnicas de tratamento e os principais métodos de controle. 2 ed. São Paulo: Lemos Editorial; 2003. 64 p.

Distl O, Kräusslich H, Mair A, Spielmann C, Diebschlag W. Computergestützte Analyse von Druckverteilungsmessungen an Rinderklauen. Dtsch tierärztl Wschr. 1990;97(11):441-508.

Ferreira PM, Carvalho AU, Facury Filho EJ, Coelho SG, Ferreira MG, Ferreira RG. Sistema locomotor dos ruminantes. Belo Horizonte: Escola de Veterinária de UFMG; 2005. 40 p.

Greenough PR, Weaver AD. Lameness in cattle. Philadelphia: Saunders; 1997. 324 p.
Hahn MV, McDaniel BT, Wilk JC. Rates of hoof growth and wear in Holstein cattle. J Dairy Sci. 1986;69(8): 2148-56.

Molina LR, Carvalho AU, Facury Filho EJ, Ferreira PM, Ferreira VCP. Prevalência e classificação das afecções podais em vacas lactantes na bacia leiteira de Belo Horizonte. Arq Bras Med Vet Zootec. 1999:51(2):149-52.

Murphy PA, Hannan J. Effects of slatted flooring on claw shape in intensively housed fattening beef cattle. 5th International Symposium on Disorders of The Ruminant Digit; 24-25 ago 1986; Dublin, Irlanda. Dublin: University College Dublin; 1986. p. 2-7.

Nicoletti JLM. Manual de podologia bovina. Barueri: Manole; 2004. $130 \mathrm{p}$.

Ollhoff RD, Ortolani EL. Comparação do crescimento e do desgaste do casco em bovinos taurinos e zebuínos. Cienc Rural. 2007;31(1):67-71.

Russel AM, Rowlands GJ, Shaw SR, Weaver AD. Survey of lameness in British dairy cattle. Vet Rec. 1982;111(8):155-60.

Silva LAF, Franco LG, Atayde IB, Cunha PHJ, Moura MI, Goulart DS. Effect of biotin supplementation on claw horn growth in young, clinically healthy cattle.

Can Vet J. 2010;51(6):607-10.

Sokal RR, Rohlf FJ. Biometry: the principles and practice of statistics in biological research. São Francisco: W. H. Freeman and Company; 1969. 776 p.

Tranter WP, Morris RS. Hoof growth and wear in pasturefed dairy cattle. N Z Vet J. 1992;40(3):89-96.

Vermunt JJ, Greenough PR. Structural characteristics of the bovine claw: horn growth and wear, horn hardness and claw conformation. Br Vet J. 1995;151(2):157-80. 